\title{
LA SIMBOLOGÍA DE LAS MÁQUINAS \\ EN LA OBRA DE ZOLA
}

\section{THE SYMBOLISM OF THE MACHINES IN THE WORK OF ZOLA}

\author{
Anna-Maria CORREDOR PLAJA \\ Universitat de Girona \\ annamaria.corredor@udg.edu
}

Resumen: Son muchos los autores que han escrito desde su pasión por la ciencia y que han reflejado en sus obras los avances de su tiempo; Zola es uno de ellos. A partir del análisis de unos cuantos textos zolianos, en los que se ha estudiado el protagonismo de las máquinas, el objetivo de este artículo es mostrar de qué manera la literatura se convierte en un instrumento al servicio de la ciencia y, al mismo tiempo, de qué manera la ciencia se convierte en materia literaria..

Palabras clave: Zola. Ciencia. Máquinas. Simbología. Siglo XIX.

Abstract: There are many authors who have written from their passion for science and who have reflected in their works the progress of their time; Zola is one of them. From the analysis of a few zolian texts, in which we have studied the role of the machines, the aim of this paper is to show how literature becomes a tool for science and, at the same time, how science becomes literary subject.

Key Words: Zola. Science. Machines. Symbolism. XIX century. 


\section{INTRODUCCIÓN}

Nacido en París en 1840, Zola vivió en primera persona los cambios político- sociales derivados de la Revolución Francesa y la difusión de las nuevas ideas morales y religiosas, pero ante todo fue un testigo de primera mano de los avances técnicos y científicos que se produjeron especialmente durante la segunda mitad del siglo XIX, y que llegaron acompañados de "I'avènement du règne de la machine" (Darcos, 1992: 320). El célebre médico y escritor Louis Figuier (Montpellier, 1819-París, 1894) escribió una bella metáfora para ilustrar el concepto de ciencia y, sobre todo, su vulgarización, la idea de que fuera accesible para todos: "La science est un soleil: il faut que tout le monde s'en approche pour se réchauffer et s'éclairer" (citado por Bensaude-Vincent, 1993: 49).

En este contexto de cambios profundos y de divulgación científica, como destaca el mismo Darcos, tres nombres influyeron profundamente sobre Zola: de Auguste Comte, el padre del positivismo científico, Zola aprende a observar los hechos sociales como si fueran fenómenos clínicos; Darwin y su teoría sobre la evolución de las especies le enseñan que la sociedad, igual que la naturaleza, obedece a leyes de lucha y de selección; finalmente, Claude Bernard, biólogo, médico y fisiólogo, fundador de la medicina experimental, hace que se interese por la biología, las enfermedades hereditarias, los condicionamientos fisiológicos (Darcos, 1992).

En este marco se inscribe la obra maestra Les Rougon-Macquart, que Zola publicó en veinte volúmenes entre 1871 y 1893 para ofrecer un panorama de la sociedad francesa durante la época del Segundo Imperio. Como Balzac en La comédie humaine, en la que se inspiró, Zola se había propuesto personificar los acontecimientos de aquellos años y las grandes transformaciones sociales que conllevaron (urbanismo parisino, aparición de los movimientos obreros y del sindicalismo, llegada del progreso, avances de la medicina, etc.). Sin embargo, el escritor va mucho más allá y pretende mostrar la interacción del hombre y de su entorno reflejando el papel que tiene la herencia en la constitución física y psicológica de los individuos. Zola tiene la habilidad de presentar a la sociedad como un gran caso patológico donde los individuos se comportan según el historial personal heredado y según el medio social en el que evolucionan: el objetivo del escritor es observar los hechos sociales como si fueran fenómenos clínicos, experimentales, a través de diferentes dramas personales. En definitiva, como señala Jean-François Chassay: “La science fait la science, la vulgarisation scientifique tente de l'expliquer, la fiction la met en scène" (Chassay, 2009: 17). En efecto, la ciencia, la técnica, el progreso en general son indisociables de la producción literaria del siglo XIX: muchos autores se hicieron eco de los avances científicos y tecnológicos y de las consecuencias de la revolución industrial, y mostraron su entusiasmo por los nuevos tiempos de una manera más o menos explícita 
(Zola, Hugo, Verne...), creando un tipo de literatura que, con estilos diversos, es un testimonio del nuevo contexto social y científico-técnico.

Las obras de Zola se pueden analizar desde diferentes puntos de vista: "L'œuvre zolienne a beau être animée par un projet de critique sociale, elle n'en est pas moins porteuse d'une dimension créatrice, symbolique et imaginaire qui dépasse les intentions explicites de l'auteur" (Vaillant, Bertrand y Régnier, 2006: 467). En el presente artículo nos hemos centrado en esta dimensión creadora y, más concretamente, en la simbología de las máquinas. "La science peut entrer en littérature par des chemins divers, plus ou moins cachés", afirma Danielle Jacquart (1996: 8). Entendemos que las máquinas son uno de estos caminos.

\section{LA MÁQUINA HUMANA}

Zola era un apasionado de las máquinas y de los mecanismos que permiten su funcionamiento', hasta tal punto que perpetúa la metáfora de la máquina humana tan en boga en el siglo XVIII': "Je suis simplement un curieux impitoyable qui voudrais démonter la machine humaine, rouage par rouage, pour voir comment le mécanisme fonctionne et arrive à produire de si étranges effets" (citado por Madeleine Ambrière, 1990: 400). Una variante de esta misma metáfora se encuentra en dos ocasiones en La Terre: "Oh! là, là, est-ce bête qu'on se fasse des machines pareilles!" (Zola, 1966b: 477), exclama uno de los personajes al oír el relato de una experiencia sexual. En el segundo caso, el autor compara a los ancianos Fouan, viejos y tan cansados que ya no sirven para nada, con "antiques machines jetées aux ferrailles" (Zola, 1966b: 480). Igualmente, en L'Assommoir, el autor insiste en su percepción del cuerpo humano como una máquina que necesita estar bien engrasada; así, el sepulturero Bazougue, que se emborracha a menudo, responde a Gervaise: "Quand l'ouvrage donne, faut bien se graisser les roues" (Zola, 1978a: 109). También del personaje Bec-Salé, el autor explica: "Il était allé boire une goutte, parce qu'il ne se sentait plus assez graissé pour attendre six heures" (Zola, 1978a: 188), y poco después añade: "la goutte de tout à l'heure lui chauffait la carcasse

1 Recordemos que el escritor pudo visitar cuatro de las cinco exposiciones universales celebradas en París durante el siglo XIX (1867, 1878, 1889 y 1900). Según Hemmings (1972), si Zola se interesó por las exposiciones, fue porque estaban orientadas hacia las nuevas invenciones técnicas y las nuevas aplicaciones de la ciencia a la industria, porque permitían constatar que el progreso avanzaba en la sociedad, y por el espíritu de idealismo humanitario que las impulsaba. Es de sobra conocido el artículo que Zola publicó en Le Figaro en el que expresa su entusiasmo por la exposición de aquel año y en el que compara París con «une énorme et puissante machine, fonctionnant à toute vapeur avec une furie diabolique» (Zola, 1867: 3). René Descartes (1596-1650) y Julien Offray de La Mettrie (1709-1751) fueron los precursores de la concepción del cuerpo humano como mecanismo que la ciencia debe encargarse de desmontar (Robles, 2009). 
comme une chaudière, il se sentait une sacrée force de machine à vapeur" (Zola, 1978a: 190). En la misma obra, el autor atribuye tanto al personaje Mes-Bottes como a Coupeau "un rire de poulie mal graissée" (Zola, 1978a: 50, 167), una expresión que se transforma en "grincement de poulie mal graissée" en Germinal (Zola, 1978b: 13), cuando el autor realiza la descripción del personaje Bonne-mort. También en la misma obra, se describe al hombre como "une machine à extraire la houille" (Zola, 1978b: 162). Y en La Fortune des Rougon, el autor presenta a los obreros de una carpintería como "des pantins articulés, avec une régularité et une sécheresse de machine" (Zola, 1960a: 8). Destacamos en este último ejemplo el uso de la palabra"pantins": los obreros se comportan como marionetas mecánicas, como si hubieran perdido su carácter humano y actuaran por voluntad ajena.

Finalmente, en Le Ventre de París, se utiliza de nuevo la imagen de la máquina de vapor para evocar el espacio bullicioso del gran mercado de abastos y su actividad incesante:

Elles [les halles] apparurent comme une machine moderne, hors de toute mesure, quelque machine à vapeur, quelque chaudière destinée à la digestion d'un peuple, gigantesque ventre de métal, boulonné, rivé, fait de bois, de verre et de fonte, d'une élégance et d'une puissance de moteur mécanique, fonctionnant là, avec la chaleur du chauffage, l'étourdissement, le branle furieux des roues (Zola, 1960c: 626).

Los ejemplos mostrados en este apartado, que ilustran la concepción del hombre como máquina, nos remiten asimismo al concepto de deshumanización, asociado a las condiciones de trabajo del proletariado, en particular durante el siglo XIX.

\section{LA FUNCIÓN DE LAS MÁQUINAS}

En los textos zolianos, las máquinas, símbolos del progreso, fascinan ${ }^{4}$, son un elemento de seducción, pero pueden asimismo representar una amenaza o un peligro, o pueden ser también un motivo de esperanza. Además, como veremos más adelante, muy a menudo los diferentes artilugios aparecen personificados y se convierten en testigos de confidencias o comparten el dolor de los personajes. Examinaremos a continuación

3 Zola recurre muy a menudo a la metáfora de la máquina de vapor, uno de los símbolos más representativos de la revolución industrial.

4 Geoff Woollen ha estudiado la frecuencia de la palabra machine en La Bête humaine y constata que ha sido utilizada en 138 ocasiones, mientras que locomotive solo aparece dos veces. En palabras de Woollen «c'est donc le tour oral qui dut fasciner Zola [...] machine est plus susceptible d'extension métaphorique que locomotive» (Woollen, 1983: 118). La seducción ejercida por las máquinas que encontramos en algunos textos se corresponde sin lugar a dudas con esta fascinación léxica señalada por Woollen. 
varios ejemplos sacados de algunas de las novelas que forman Les Rougon-Macquart, que ilustran las diferentes funciones atribuidas a las máquinas.

\subsection{LA MÁQUINA COMO SÍMBOLO DEL PROGRESO}

La Terre se presta especialmente a esta interpretación. La novela empieza con la descripción de una escena en el campo. El protagonista, Jean, se encuentra sembrando un campo manualmente: "Jean, ce matin-là, un semoir de toile bleue noué sur le ventre, en tenait la poche ouverte de la main gauche, et de la droite, tous les trois pas, il y prenait une poignée de blé, que d'un geste, à la volée, il jetait" (Zola, 1966b: 367). Sin embargo, en el segundo párrafo se explica que una sembradora mecánica hace lo mismo en otro lugar: "La parcelle de terre, d'une cinquantaine d'ares à peine, au lieu-dit des Cornailles, était si peu importante, que M. Hourdequin, le maître de la Borderie, n'avait pas voulu y envoyer le semoir mécanique, occupé ailleurs" (Zola, 1966b: 367). Observamos que la sembradora mecánica se reserva para el campo más importante, por consiguiente se está dando más valor al trabajo mecánico que al humano. A lo largo de la novela, el autor insiste en mostrar el paralelismo entre el trabajo mecánico y el manual; así, en el capítulo cuarto de la segunda parte, leemos: "La ferme n'ayant pas de machine à faner, on lui avait laissé engager deux faneuses, Palmyre [...] et Françoise" (Zola, 1966b: 475); en el capítulo cuarto de la tercera parte, a falta de una segadora mecánica, se recurre a un grupo de trabajadores: "L'année d'auparavant, sa moissonneuse mécanique s'était détraquée; et, désespéré du mauvais vouloir de ses serviteurs, arrivant à douter lui-même de l'efficacité des machines, il avait dû se précautionner d'une équipe de moissonneurs, dès I'Ascension" (Zola, 1966b: 564). Es evidente que el autor, en un intento de conciliar las diferentes reacciones, a menudo antagónicas, ante la mecanización agrícola, quiere expresar la idea de que el uso de los nuevos artilugios puede coexistir con la pervivencia de las prácticas tradicionales.

El señor Hourdequin encarna en La Terre al gran propietario con nuevas ideas para modernizar el campo con la introducción de abonos químicos y maquinaria agrícola, y por esta razón es objeto de burla, ya que los campesinos "ricanaient de ses machines" (Zola, 1966b: 441). Hourdequin representa los nuevos tiempos, el progreso que llega lentamente ante el escepticismo y la actitud hostil de la gente del campo:

Les uns, comme moi, sont pour la grande [propriété], parce qu'elle paraît aller dans le sens même de la science et du progrès, avec l'emploi de plus en plus large des machines, avec le roulement des gros capitaux... Les autres, au contraire, ne croient qu'à l'effort individuel et préconisent la petite (Zola, 1966b: 492-493). 
Una frase como: "Une seule machine, la machine à battre, commençait à être acceptée" (Zola, 1966b: 490) expresa claramente este escepticismo rural y la lentitud con la que se introduce la mecanización. Precisamente el autor menciona a menudo la "batteuse à vapeur [...] la machine, soufflant du matin au soir, faisant voler au soleil une poussière blonde, emplissait le pays d'un ronflement énorme et continu" (Zola, 1966b: 590). El zumbido de este artilugio que, imparable, se extiende por todo el país, bien puede interpretarse como el símbolo del progreso. En el capítulo sexto de la tercera parte, el autor vuelve a destacar este zumbido que, persistente, se propaga por todas partes: "au milieu du grand silence, on entendait le ronflement lointain de la batteuse à vapeur, qui ne cessait pas" (Zola, 1966b: 598).

\subsection{LA MÁQUINA COMO OBJETO DE SEDUCCIÓN}

En los textos zolianos, el funcionamiento de las máquinas seduce a menudo a los personajes: en el primer capítulo de La Terre, Jean y Françoise observan la sembradora llenos de curiosidad: "À leur droite, ils eurent un regard pour un semoir mécanique, dont les chevaux tournèrent près d'eux" (Zola, 1966b: 371). Destacamos que los personajes no ven la máquina, sino que la miran, como un objeto raro y fascinante.

Observamos esta misma fascinación en L'Assommoir, cuando Gervaise quiere contemplar el alambique del tío Colombe: "elle eut la curiosité d'aller regarder, au fond, derrière la barrière de chêne, le grand alambic de cuivre rouge" (Zola, 1978a: 50). Y el utensilio atrae también a otros personajes: "Mes-Bottes, accompagné de ses deux camarades, était venu s'accouder sur la barrière [...] les yeux attendris, fixés sur la machine à soûler" (Zola, 1978a: 50). Pero Gervaise también se extasía contemplando su nueva máquina para planchar. En el fragmento que citamos a continuación, destacamos el uso del verbo "s'asseoir" (Gervaise se sienta para observar mejor), y de las expresiones "couver des yeux" (comerse con los ojos) y "se mettre à genoux" (el personaje se arrodilla ante el nuevo aparato como si fuera un objeto sagrado):

Gervaise s'asseyait sur un tabouret [...] couvant des yeux ses outils neufs. Mais son premier regard allait toujours à sa mécanique, un poêle de fonte, où dix fers pouvaient chauffer à la fois, rangés autour du foyer, sur des plaques obliques. Elle venait se mettre à genoux, regardait avec la continuelle peur que sa petite bête d'apprentie ne fit éclater la fonte, en fourrant trop de coke (Zola, 1978a: 150).

Una seducción similar se produce cuando, antes de regresar a casa, Gervaise se detiene para observar "le flamboiement d'une forge", en la que "toute la journée, les 
marteaux sonnaient sur l'enclume, des incendies d'étincelles éclairaient l'ombre blafarde de la cour" (Zola, 1978a: 154-155). Igualmente, Gervaise experimenta una admiración profunda por el funcionamiento de toda la maquinaria que Goujet le enseña dentro de la herrería: "elle éleva les yeux, elle s'arrêta à regarder les courroies, les longs rubans qui tendaient au plafond une gigantesque toile d'araignée [...] dont elle regardait, les yeux levés, la force énorme et muette passer dans la nuit vague des charpentes" (Zola, 1978a: 194-195). Esta admiración aumenta a medida que Goujet le va mostrando los diferentes artilugios:"Il passa aux machines: les cisailles mécaniques [...], les machines à boulons et à rivets [...], les ébarbeuses [...] les taraudeuses..." (Zola, 1978a: 194). Es evidente que el autor se complace en enumerar los diferentes utensilios de la herrería 5 .

En La Bête humaine ${ }^{6}$, observamos en el primer capítulo cómo Roubaud, el subjefe de estación, desde una ventana de un quinto piso contempla absorto la actividad de los trenes de una estación parisina:

II suivit des yeux la machine de manœuvre, une petite machine-tender, aux trois roues basses et couplées, qui commençait le débranchement du train, alerte besogneuse, emmenant, refoulant les wagons sur les voies de remisage. Une autre machine, puissante celle-là, une machine d'express... (Zola, 1966a: 998).

En Germinal, en el tercer capítulo de la primera parte, antes de describir la máquina de extracción de la mina, el autor señala que Étienne se siente "attiré par la machine, dont il voyait maintenant luire les aciers et les cuivres" (Zola, 1978b: 27). Al principio del capítulo sexto, el autor menciona la máquina, que es observada de nuevo por Étienne, y que destaca en un ambiente de lo más sórdido: "Ce n'était que nu et sale. Un jour terreux entrait par les fenêtres poussiéreuses. Seule, la machine luisait, là-bas, avec ses cuivres..." (Zola, 1978b: 63). Encontramos una descripción similar en el primer capítulo de la tercera parte, cuando el brillo de la máquina destaca en medio de la oscuridad (Zola, 1978b: 131), y también en el capítulo tercero de la última parte, cuando el artilugio sigue reluciendo en medio del desastre (Zola, 1978b: 453). En Germinal, el autor realiza una comparación

5 El uso del vocabulario técnico es una de las características que Zola heredó del movimiento realista. También Victor Hugo, además de ser uno de los autores más representativos del romanticismo francés, mostró su pasión por la ciencia y la técnica muy especialmente en su novela Les travailleurs de la mer, en la que escribe largas enumeraciones de términos pertenecientes al léxico del mar, de la industria naval, de la botánica y de la zoología.

6 Según Jean Borie, en esta novela, la bestia humana representa al mismo tiempo la bestialidad inherente al hombre, y la locomotora, la máquina inventada por el hombre que se convierte en un monstruo (Borie, 1971). 
implícita de la máquina con una mujer: el aspecto de la máquina atrae y seduce a Étienne como lo haría el aspecto físico de una mujer.

\subsection{LA MÁQUINA COMO SÍMBOLO DE AMENAZA}

Como hemos señalado anteriormente, en La Terre se hace burla del propietario impulsor de la mecanización de las tareas agrícolas, y las mismas máquinas son tratadas como objetos diabólicos; así, ante una recolectora de forraje, uno de los mozos del señor Hourdequin comenta a tres campesinos del lugar: "Hein! disait-il, en voilà, un sabot!... Et ça casse l'herbe, ça l'empoisonne. Ma parole! II y a trois moutons déjà qui en sont morts" (Zola, 1966b: 495). En el mismo párrafo, observamos que la recolectora mecánica es comparada con "une bête farce et méchante". En el fondo, las máquinas, "inventions du diable contre le pauvre monde", dan miedo porque se perciben como un peligro: "Qu'est-ce qu'elles feront, nos femmes, si l'on se passe d'elles, aux foins?" (Zola, 1966b: 495) se pregunta uno de los campesinos. Por eso el mozo de Hourdequin insulta a la máquina dándole una patada y diciendo: "Hue donc, carcasse!" (Zola, 1966b: 495). Por consiguiente, las máquinas son una amenaza porque harán el trabajo humano, pero también por el coste que, junto con otros avances, representan:

—Un homme de progrès, un homme de progrès, répéta Hourdequin de son air gaillard, sans doute j'en suis un; mais ça me coûte si cher, que je vais bientôt ne plus pouvoir me payer ce luxe... Les machines, les engrais chimiques, toutes les méthodes nouvelles, voyez-vous, c'est très beau, c'est très bien raisonné, et ça n'a qu'un inconvénient, celui de vous ruiner d'après la saine logique.

-Parce que vous êtes un impatient, parce que vous exigez de la science des résultats immédiats, complets, parce que vous vous découragez des tâtonnements nécessaires, jusqu'à douter des vérités acquises et à tomber dans la négation de tout! (Zola, 1966b: 679).

En esta conversación de Hourdequin con un librecambista observamos el desánimo del propietario que se ve abocado a invertir grandes cantidades de dinero en la modernización de los cultivos, mientras que su interlocutor deja entrever que los adelantos se introducen lentamente y que se requiere paciencia para constatar las mejoras: son dos puntos de vista antagónicos, que representan dos maneras diferentes de entender el progreso. 
Igualmente, el herrero Goujet, en L'Assommoir, es consciente del poder de los nuevos artilugios, y, como a los mozos de Hourdequin, le dan ganas de golpearlos cuando constata que son superiores a los hombres':

En douze heures, cette sacrée mécanique en fabriquait des centaines de kilogrammes. Goujet n'avait pas de méchanceté; mais, à certains moments, il aurait volontiers pris Fifine pour taper dans toute cette ferraille, par colère de lui voir des bras plus solides que les siens. Ça lui causait un gros chagrin, même quand il se raisonnait, en se disant que la chair ne pouvait pas lutter contre le fer. Un jour, bien sûr, la machine tuerait l'ouvrier; déjà leurs journées étaient tombées de douze francs à neuf francs, et on parlait de les diminuer encore; enfin, elles n'avaient rient de gai, ces grosses bêtes... (Zola, 1978a: 195).

Goujet llega incluso a pensar que las máquinas, como auténticas rivales, pueden poner en peligro su amistad con Gervaise: "un moment il avait eu peur qu'elle ne le méprisât, après avoir vu les machines" (Zola, 1978a: 196). También en el capítulo noveno, otro personaje, la madre de Goujet, se lamenta: "On avait encore baissé la journée des boulonniers; de neuf francs, elle était tombée à sept francs, à cause des machines qui, maintenant, faisaient toute la besogne" (Zola, 1978a: 317). A través de los comentarios de los Goujet, el autor alude directamente a uno de los efectos negativos de la revolución industrial: la baja generalizada de los salarios.

En la misma obra, como hemos visto anteriormente, el alambique del tío Colombe es un artilugio que seduce, pero al mismo tiempo tiene un carácter diabólico. En el capítulo segundo, el alambique es tratado de "cuisine du diable", expresión que el autor transforma en "cuisine d'enfer" en el capítulo décimo. El utensilio es presentado como un personaje maléfico, capaz de provocar a Gervaise "la sensation d'un malaise plus inquiétant derrière son dos" (Zola, 1978a: 389), o de hacer que sienta "un frisson dans les épaules, une peur mêlée d'un désir" (Zola, 1978a: 391). El alambique es un elemento indisociable de la historia de Gervaise y Coupeau. Al principio de la novela, Gervaise lo percibe como un mal presagio: “Gervaise, prise d'un frisson, recula [...] C'est bête, ça me fait froid, cette machine" (Zola, 1978a: 50), pero el aparato es el testigo indiscutible de la amistad que empieza entre los dos personajes, hasta que su relación amorosa y sus vidas se degradan; en ese momento Gervaise culpa abiertamente aquel artefacto: "Et, de loin, elle contemplait la machine à soûler, en sentant que son malheur venait de là"

7 La violencia de algunos personajes contra las máquinas no deja de ser una alusión al /udismo, el movimiento de rebelión social que tuvo su origen en Gran Bretaña en 1811, cuando los obreros protestaron contra la mecanización de la industria textil destruyendo la nueva maquinaria. 
(Zola, 1978a: 464). Así, esta reflexión final de Gervaise nos retorna a la primera visión del alambique como un artilugio fascinante y amenazador.

En Le Ventre de Paris, los diferentes objetos de la tienda de Quenu también son testigos de los acontecimientos turbios que allí tienen lugar: "plusieurs appareils, fixés sur le bloc, une pompe à injecter, une machine à pousser, une hacheuse mécanique, mettaient là, avec leurs rouages et leurs manivelles, l'idée mystérieuse et inquiétante de quelque cuisine de l'enfer" (Zola, 1960c: 682). Y en la misma obra, cuando Lise quiere indagar sobre la vida misteriosa de su cuñado Florent, el autor escribe: "elle s'imaginait des choses terribles, une machine infernale fabriquée en haut" (Zola, 1960c: 788); observamos pues que se recurre a la idea de la máquina como un objeto perverso.

Asimismo, en Germinal el complejo minero del Voreux se presenta ya en el primer capítulo como un lugar inquietante cuando el autor compara su chimenea con "une corne menaçante" (Zola, 1978b: 9). Igualmente en La Terre, destacamos este comentario de Jean: "Faudrait pas trop songer à ces machines-là, on en deviendrait fou” (Zola, 1966b: 687), que tiene una connotación claramente negativa, ya que equivale a decir que no se puede esperar nada bueno de la nueva maquinaria agrícola.

Jean Borie señala que una de las características de las machines-monstres, que aparecen de manera recurrente en los relatos de Zola, es que desprenden agresividad (Borie, 1971). En efecto, como veremos más adelante, las máquinas zolianas gritan, chillan, devoran, galopan, como si fueran auténticas fieras: la máquina 608 y sus dieciocho vagones, es decir, el tren fantasma de La Bête humaine, es un buen ejemplo de ello.

\subsection{LA MÁQUINA COMO SÍMBOLO DE ESPERANZA}

Asociadas al progreso, las máquinas representan un futuro mejor. Uno de los personajes de La Terre, simpatizante de las ideas socialistas, afirma: "les machines travailleront pour nous, la journée de simple surveillance ne sera plus que de quatre heures; peut-être même qu'on arrivera à se croiser complètement les bras" (Zola, 1966b: 686). En este caso, los nuevos artilugios no se consideran una amenaza para el hombre, sino un alivio, una mejora notable de sus condiciones de vida. En el fragmento siguiente, se percibe incluso la idea del dominio del hombre sobre la máquina:

[...] I'homme simplement chargé de la surveillance, tout le travail fait par les machines, charrues doubles armées de disques tranchants, semoirs et sarcloirs, moissonneuses-lieuses, batteuses locomobiles avec élévateur de paille et ensacheur; des paysans qui sont des mécaniciens, un peloton d'ouvriers suivant à cheval chaque machine, toujours prêts à descendre serrer un écrou, changer un boulon, forger une pièce (Zola, 1966b: 767). 
Igualmente, en L'Assommoir, el herrero Goujet, después de hablar mal de las máquinas, añade: "Hein ! Ça nous dégotte joliment ! Mais peut-être que plus tard ça servira au bonheur de tous" (Zola, 1978a: 196). A través de las palabras de Goujet, observamos que Zola hace una clara alusión a las ideas de Robert Owen, uno de los padres del socialismo.

Las máquinas acompañan a los personajes en los acontecimientos de sus vidas y, en determinadas situaciones, el autor las convierte en buenos presagios. Así, en el capítulo sexto de L'Assommoir, cuando Gervaise toma la decisión de alquilar un local para instalarse como planchadora, el autor recurre a la imagen de un aparato en funcionamiento y al ruido de las herramientas de una carpintería próxima para expresar la emoción de aquel momento. Además, la visión de las aguas verdes procedentes de la tintorería se añade a la alegría de la protagonista, que las interpreta como un buen augurio ${ }^{8}$ :

Il lui semblait faire quelque chose de très hardi, se jeter au beau milieu d'une machine en branle, pendant que les marteaux du serrurier et les rabots de l'ébéniste tapaient et sifflaient au fond des ateliers du rez-de-chaussée. Ce jour-là, les eaux de la teinturerie coulant sous le porche étaient d'un vert pomme très tendre. Elle les enjamba, en souriant, elle voyait dans cette couleur un heureux présage (Zola, 1978a: 146).

Una situación similar se encuentra en el mismo capítulo, cuando Gervaise, que cada vez se siente más atraída por Goujet, cada viernes encuentra un pretexto para hacerle una visita en su taller; a partir de entonces la herrería se convierte en el testigo mudo de su relación:

Dès qu'elle tournait le coin de la rue, elle se sentait légère, gaie, comme si elle faisait une partie de campagne [...] son cour sautait à la danse des marteaux [...] Goujet l'attendait, les bras nus, la poitrine nue, tapant plus fort sur l'enclume, ces jours-là, pour se faire entendre de plus loin [...] pendant un printemps, leurs amours emplirent ainsi la forge d'un grondement d'orage (Zola, 1978a: 214-215).

En La Bête humaine, en el capítulo noveno, encontramos un episodio en el que Lantier explica a Séverine que uno de sus compañeros se marcha a Nueva York para "exploiter une invention nouvelle, une machine à fabriquer des boutons" (Zola, 1966a: 1233) y le

8 Como señala Veloso Santamaría, los textos zolianos "rebosan de impresiones sensoriales que, lejos de limitarse a una función informativa, son la clave para penetrar en un universo de símbolos, más allá de la realidad percibida" (Veloso Santamaría, 2012: 123). En este sentido, la misma autora destaca la importancia de la luz y de los colores en la obra de Zola, a quien relaciona con los pintores impresionistas. 
ha planteado la posibilidad de acompañarle como socio en el negocio. En este caso, el artilugio recién inventado es el punto de partida de un sueño: "Nous partirions demain" (Zola, 1966a: 1233) le responde Séverine, utilizando el condicional, ya que para poder viajar necesitan deshacerse de Roubaud, y en un instante ella imagina la nueva vida que podrían empezar juntos "loin de tout souvenir, rien que des jours nouveaux devant nous!" (Zola, 1966a: 1233).

En La Curée, Saccard utiliza la imagen del alambique para expresar su admiración por la transformación urbanística que se está produciendo en París: “On dirait que le quartier bout dans l'alambic de quelque chimiste" (Zola 1960b: 388); en este caso, el alambique, como si fuera un elemento mágico, representa la esperanza del cambio. En la misma obra, aparece también la expresión "une machine à pièces de cent sous", e igualmente en plural, "les machines à pièces de cent sous" (Zola 1960b: 392, 394), para designar las operaciones especuladoras con las que se enriquecen Saccard y otros personajes: para ellos, inventar una de esas máquinas equivale a asegurarse una vida holgada?

\subsection{LA MÁQUINA PERSONIFICADA}

El manual de las figuras del discurso de Pierre Fontanier propone esta definición de la personificación: "La personnification consiste à faire d'un être inanimé, insensible, ou d'un être abstrait et purement idéal, une espèce d'être réel et physique, doué de sentiment et de vie, enfin ce qu'on appelle une personne" (Fontanier, 1977: 111). En las obras de Zola hay numerosos ejemplos que ilustran esta definición, y que permiten observar el comportamiento de las máquinas como personas, tanto por sus características físicas como por los sentimientos que expresan.

Así, en Le Ventre de París, "les broches finissaient par causer entre elles, par adresser des mots aimables à Quenu" (Zola, 1960c: 643). Hemos citado anteriormente el zumbido de la trilladora de La Terre; el autor se sirve de una comparación y personifica este artilugio cuando, para expresar la tristeza del protagonista después de una pelea involuntaria, escribe: "Le ronflement de la machine, au fond du crépuscule, se prolongeait comme une grande plainte de détresse" (Zola, 1966b: 606).

También en L'Assommoir, leemos que Gervaise "traversait le pont du chemin de fer, dans le branle des trains, grondant et déchirant l'air du cri désespéré de leurs sifflets" (Zola, 1978a: 466). En La Bête humaine, la locomotora lanza "un long cri de plainte" (Zola, 1966a: 1168), mientras otra máquina "sifflait, jetant à la nuit une plainte de mélancolique détresse" (Zola, 1966a: 1239).

9 El dinero, la especulación, las finanzas, el juego aparecen de manera recurrente en la literatura del siglo XIX. Zola se hizo eco de la importancia social que adquirió el dinero durante el siglo XIX, de la misma manera que lo hicieron Balzac, Maupassant, Villiers de I'Isle-Adam, Mérimée, Musset, entre otros. 
En L'Assommoir, en el episodio del lavadero, la máquina de vapor "semblait hausser la voix, vibrante, ronflante, emplissant l'immense salle" (Zola, 1978a: 26). Unas páginas más adelante, el mismo artilugio se describe como "haletante, fumante, secouant plus rudement le lavoir de la besogne continue de ses bras d'acier" (Zola, 1978a: 39). Se trata pues de una mecanismo vivo. Y también está vivo el alambique del tío Colombe, que el autor describe como si fuera un personaje de carne y huesos (fijémonos en el uso de las palabras mine, souffle, ronflement, travailleur morne, gaiété, sueur):

L'alambic [...] gardait une mine sombre [...] à peine entendait-on un souffle intérieur, un ronflement souterrain; c'était comme une besogne de nuit faite en plein jour, par un travailleur morne, puissant et muet [...] L'alambic, sourdement, sans une flamme, sans une gaieté dans les reflets éteints de ses cuivres, continuait, laissait couler sa sueur d'alcool (Zola, 1978a: 50).

En Germinal, el autor explica que las instalaciones del Voreux tienen "un mauvais air de bête goulue, accroupie là pour manger le monde" (Zola, 1978b: 9), y a lo largo de la novela el autor insiste en llamar a la mina "bête méchante" (Zola, 1978b: 17) o "bête mauvaise" (Zola, 1978b: 130, 436), o “bête lasse et repue" (Zola, 1978b: 362); en definitiva, se trata de una bestia que respira y digiere carne humana (Zola, 1978b: 17), que se traga a los hombres a bocados (Zola, 1978b: 29). Asimismo, la jaula de hierro que sirve para transportar a los hombres es comparada con una "bête nocturne" (Zola, 1978b: 29) o con una "bête de nuit" (Zola, 1978b: 34), y el pozo de la mina es igualmente personificado cuando el autor lo llama "le monstre" (Zola, 1978b: 362, 493), el monstruo que devora las cargas de hombres "sans un arrêt, toujours affamé, de boyaux géants capables de digérer un peuple" (Zola, 1978b: 29-30), una información que vuelve a repetirse al final del capítulo tercero: "Le puits dévorateur avait avalé sa ration quotidienne d'hommes" (Zola, 1978b: 39), y sobre la que se insiste en el capítulo séptimo de la cuarta parte: “Ces misérables, on les jetait en pâture aux machines" (Zola, 1978b: 277). Cuando la mina de Jean-Bart estalla y el encargado Deneulin toca el metal frío de la máquina, leemos: "le froid lui donna un frisson, comme s'il avait touché un mort" (Zola, 1978b: 312). Con la personificación de la mina, en Germinal el autor añade dramatismo a la explotación de la clase obrera (viejos, adultos y menores, hombres y mujeres indistintamente) y a la creciente rebelión de los mineros contra el sistema capitalista.

A lo largo de las narraciones, se constata el interés de Zola por transmitir la idea de que las máquinas son entidades vivas, y por eso describe su funcionamiento utilizando y repitiendo verbos como ronfler, souffler, s'essouffler, s'épuiser, râler, siffler, secouer, remuer, se cabrer, avaler, engloutir, boire, manger, croquer, dévorer, cracher, se cabrer; sustantivos como ronflement, râle, souffle, hoquet, sifflet, sifflement, cri (aigre, aigu, d'agonie), essoufflement, 
roulement, secousses, haleine; adjetivos como gentille, docile, rétive, courageuse, douce, obéissante, endormie, froide, haletante, lasse, repue, essoufflée, affolée, quinteuse, revêche, dévorateur, dévorante, fainéante, agonisante. El autor menciona muy a menudo l'âme de las máquinas, e insiste mucho en la palabra haleine: "I'haleine régulière de la machine à vapeur" (Zola, 1978a: 115), "I'haleine forte de la scierie mécanique" (Zola, 1978a: 289), "l'essoufflement de son haleine" (Zola, 1978a: 463), "I'haleine chaude" (Zola, 1966a: 1050), "hors d'haleine" (Zola, 1966a: 1170), "reprendre haleine" (Zola, 1966a: 1172), "d'une haleine éperdue" (Zola, 1966a: 1173), "haleine brûlante" (Zola, 1966a: 1250), "une haleine blanche" (Zola, 1966a: 1260), "le bruit strident de son haleine" (Zola, 1966a: 1330), "cette haleine immense" (Zola, 1966a: 1267), "haleine plus grosse et plus longue" (Zola, 1978b: 17). Observamos la insistencia en el aliento, pero también en le sang de las máquinas: "elle revenait, à la vie, au sang même des machines" (Zola, 1978a: 195), y sobre todo en la visión de la máquina como persona: "On vit la machine, disloquée sur son massif, les membres écartelés, lutter contre la mort : elle marcha, elle détendit sa bielle, son genou de géante, comme pour se lever; mais elle expirait, broyée, engloutie" (Zola, 1978b: 454).

Una de las personificaciones más conocidas de Zola es la de la Lison, la locomotora de La Bête humaine. De hecho, ya en el primer capítulo (Zola, 1966a: 998), se produce una especie de diálogo muy particular en el que un tren espera a su máquina, la máquina "en personne que l'impatience gagne" solicita la vía, la máquina responde a una señal que había entendido... Esta personificación está presente a lo largo de la narración; en este mismo capítulo, por ejemplo, las máquinas de maniobras son comparadas con "des ménagères vives et prudentes" (Zola, 1966a: 1010). Pero es la personificación de la Lison lo que realmente llama la atención, porque no se trata de una personificación puntual sino que se mantiene a lo largo de la novela. En el capítulo quinto, el autor atribuye la personificación de la máquina al personaje de Jacques Lantier, y lo explica así:

Ainsi que les autres machines de la Compagnie de l'Ouest, en dehors du numéro qui la désignait, elle portait le nom d'une gare, celui de Lison, une station du Cotentin. Mais Jacques, par tendresse, en avait fait un nom de femme, la Lison, comme il disait, avec une douceur caressante.

Et, c'était vrai, il l'aimait d'amour, sa machine [...] il n'ignorait point que chacune avait son caractère, que beaucoup ne valaient pas grand'chose, comme on dit des femmes de chair et d'os; de sorte que, s'il l'aimait celle-là, c'était en vérité qu'elle avait des qualités rares de brave femme (Zola, 1966a: 1128). 
Por consiguiente, el mismo personaje es quien crea la personificación10, en este caso de la máquina de tren. Este fenómeno se repite en otras obras: en L'Assommoir es la misma Gervaise quien en una ocasión personifica al alambique, cuando el autor escribe que sentía: "des envies de sauter sur le grand alambic comme sur une bête, pour le taper à coups de talon et lui crever le ventre. Tout se brouillait, elle voyait la machine remuer, elle se sentait prise par ses pattes de cuivre..." (Zola, 1978a: 393). Sin embargo, la relación de Gervaise con el alambique poco tiene que ver con la de Jacques Lantier y la Lison. Asimismo, en Germinal los obreros producen la personificación cuando se lanzan furiosos sobre la bomba de la máquina de extracción: "On se jetait sur elle [la pompe] comme sur une personne vivante, dont on voulait la vie" (Zola, 1978b: 322).

En cuanto a la Lison, hay que destacar que el uso del artículo femenino delante del nombre propio contribuye a hacerlo más próximo, más familiar, como si se atribuyera realmente a una amante de Jacques Lantier. En este sentido, hay que destacar el uso de un campo léxico con una clara connotación erótica: la máquina es una metáfora de la mujer. Lantier lucha contra la pulsión de muerte, que aparece asociada a la pulsión sexual cada vez que mira a una mujer. En efecto, la Lison es comparada con una "maîtresse apaisante, dont il n'attendait que du bonheur" (Zola, 1966a: 1044), o con una"femme vieillie et moins forte" (Zola, 1966a: 1164), o con "ces femmes jeunes, solidement bâties" (Zola, 1966a: 1187), o con una "personne pressée qui accourt" (Zola, 1966a: 1257). Es particularmente interesante el episodio del accidente de la Lison en la nieve (capítulo séptimo), preludio del segundo accidente, fatal, que provoca su destrucción definitiva (capítulo décimo); una vez más, la Lison es tratada como una mujer: "lui qui la connaissait à fond, venait de la sentir singulière sous sa main, changée, vieillie, touchée quelque part d'un coup mortel" (Zola, 1966a: 1187). Como cualquier mujer, la Lison se adormece, sopla, resopla, gime, se agota, envejece, sufre heridas antes de morir: "c'était la fin, la secousse de l'agonie [...] la Lison s'arrêta définitivement, expirant, dans le grand froid. Son souffle s'éteignit, elle était immobile, et morte" (Zola, 1966a: 1173). Cuando se produce el accidente fatal, leemos:"La pauvre Lison n'en avait que pour quelques minutes [...] [elle] prenait l'affreuse tristesse d'un cadavre humain" (Zola, 1966a: 1266-1267). Incluso cuando la máquina es destruida, el autor hace esta reflexión: "C'était donc fini, leur ménage à trois?" (Zola, 1966a: 1267), porque la Lison es el eslabón que une la relación de Jacques y Séverine, pero también la de Jacques y Pecqueux.

Una vez muerta la Lison, aparece una nueva máquina, que vuelve a ser personificada; así, el autor menciona su "pucelage" (Zola, 1966a: 1301), o "les caprices, les écarts de

10 Victor Hugo utiliza este mismo tipo de personificación en Les travailleurs de la mer: mess Lethierry bautiza su barco de vapor con un nombre femenino, la Durande, y lo equipara así a su sobrina Déruchette; si Déruchette es la hija que nunca ha tenido, la Durande es la metáfora de la mujer con la que nunca se ha casado. 
jeunesse [...] il la sentait rétive, fantasque" (Zola, 1966a: 1328), pero esta segunda máquina pronto se transforma en un monstruo, una bestia desenfrenada: "C'était le galop tout droit, la bête qui fonçait tête basse et muette, parmi les obstacles. Elle roulait, roulait sans fin, comme affolée de plus en plus par le bruit strident de son haleine" (Zola, 1966a: 1330). El paso acelerado de esta segunda máquina puede interpretarse, al igual que el zumbido persistente de la trilladora en La Terre comentado anteriormente, como un símbolo del progreso que avanza imparable, salvando todos los obstáculos.

\section{CONCLUSIONES}

Hemos expuesto en este trabajo diversos ejemplos que ilustran la pasión de Zola por las máquinas y el carácter simbólico que adquieren en su obra. Hemos interpretado las máquinas como objetos que fascinan, como símbolos de esperanza y de progreso, pero también como símbolos de amenaza. El uso de la figura retórica de la personificación, un elemento recurrente en los textos de Zola, nos ha permitido constatar cómo el autor se ha servido hábilmente de los recursos literarios para expresar, magnificándola, su pasión en el texto. ¿Podemos imaginar cómo serían las novelas de Zola sin el recurso de la personificación? Es decir, ¿podemos imaginar La Bête humaine con una locomotora llamada Lison pero tratada como una simple máquina de tren, o L'Assommoir con un alambique que solo es una caldera, algunos tubos y un depósito? Haciendo una especie de oxímoron, Zola tiene el acierto de mostrar la imagen seductora de la ciencia y de la técnica y sus amenazas y peligros, y lo hace partiendo de su sensibilidad hacia el ámbito científico en general, pero sobre todo desde la literatura. Si, como afirma Jean-François Chassay, la ficción permite ver la evolución de la ciencia desde el interior y en un marco social singular (Chassay, 2009), después de analizar una parte de la obra de Zola, no podemos sino acabar citando esa afirmación rotunda: "Émile Zola es el siglo XIX" (Veloso Santamaría, 2012: 5).

\section{REFERENCIAS BIBLIOGRÁFICAS}

AMBRIÈRE, M. (sous la direction de) (1990). Précis de littérature française du XIXe siècle. Paris: Presses Universitaires de France.

BENSAUDE-VINCENT, B. (1993). “Un public pour la science: l'essor de la vulgarisation au XIXe siècle". Réseaux 11.58, 47-66 (también en: http://enssibal.enssib.fr/autres-sites/reseauxcnet/58/03-bensa.pdf[11/01/2016]).

BORIE, J. (1971). Zola et les mythes, ou de la nausée au salut. París: Seuil.

CHASSAY, J.-F. (2009). Si la science m'était contée: des savants en littérature. París: Seuil.

DARCOS, X. (1992). Histoire de la littérature française. París: Hachette. 
FONTANIER, P. (1977). Les figures du discours. París: Flammarion.

HEMMINGS, F. W. (1972). "Emile Zola devant I'Exposition Universelle de 1878". Cahiers de I'Association Internationale d'Études Françaises 24.24, 131-153, http://www.persee.fr/web/ revues/home/prescript/article/caief_05715865_1972_num_24_1_1005 [10/01/2016].

JACQUART, D. (1996). "Avant-propos". En De la science en littérature à la science-fiction: actes du 119 e congrès national des sociétés historiques et scientifiques, D. Jacquart (ed.), 7-10. París: Éditions du C.T.H.S.

ROBLES, F. (2010). “Du mécanique plaqué sur du vivant: images de l'Africain-machine dans les discours scientifiques et littéraires occidentaux, XVIIle-XXe siècles". Épistémocritique 7, http://www.epistemocritique.org/spip.php?article171\&lang=fr9 [09/01/2016].

VAILLANT, A.; BERTRAND, J.-P. \& RÉGNIER, Ph. (2006). Histoire de la littérature française du XIXe siècle. Rennes: Presses Universitaires de Rennes.

VELOSO SANTAMARÍA, I. (2012). Conocer a Émile Zola. Madrid: Ediciones del Laberinto.

WOOLLEN, G. (1983). "Zola: la machine en tous ses effets". Romantisme 13.41, 115-124.

ZOLA, É. (1867). “Dans Paris. Les nids". Le Figaro, 15/05, 3.

(1960a). Les Rougon-Macquart. Histoire naturelle et sociale d'une famille sous le Second Empire. La Fortune des Rougon. París: Gallimard, "Bibliothèque de la Pléiade", vol. I.

(1960b). Les Rougon-Macquart. Histoire naturelle et sociale d'une famille sous le Second Empire. La Curée. París: Gallimard, "Bibliothèque de la Pléiade", vol. I.

(1960c). Les Rougon-Macquart. Histoire naturelle et sociale d'une famille sous le Second Empire. Le Ventre de París. París: Gallimard, "Bibliothèque de la Pléiade", vol. I.

(1966a). Les Rougon-Macquart. Histoire naturelle et sociale d'une famille sous le Second Empire. La Bête humaine. París: Gallimard, "Bibliothèque de la Pléiade", vol. IV.

(1966b). Les Rougon-Macquart. Histoire naturelle et sociale d'une famille sous le Second Empire. La Terre. París: Gallimard, “Bibliothèque de la Pléiade”, vol. IV.

(1978a). L'Assommoir. París: Fasquelle, “Le Livre de Poche”.

(1978b). Germinal. París: Fasquelle, "Le Livre de Poche”.

Recibido el 14 de febrero de 2016.

Aceptado el 15 de noviembre de 2016. 
